\title{
The Effects of Action Observation with Functional Electrical Stimulation on Corticomuscular Coherence
}

\author{
Ji Young Kim', Young Uk Ryu², Jiwon Park² \\ 'Department of Physical Therapy, Masan University, Masan, Republic of Korea; ${ }^{2}$ Department of Physical Therapy, College of Bio and Medical Science, \\ Daegu Catholic University, Gyeongsan, Republic of Korea
}

Objective: To investigate the action observation effects of functional electrical stimulation (FES) on the communication between motor cortex and muscle through corticomuscular coherence (CMC) analysis.

Methods: Electroencephalogram (EEG) and electromyogram (EMG) of 27 healthy, nonathlete subjects were measured during action observation, FES, and action observation with FES, which lasted for 7sper session for 10 times. All trials were repeated for 30 times. Simultaneously measured EEG raw data and rectified EMG signals were used to calculate CMC. Only confidence limit values above 0.0306 were used for analysis. CMC was divided into three frequency domains, andthe grand average coherence and peak coherence were computed. Repeated ANOVA was performed to analyze the coherence value difference for each condition's frequency band.

Results: CMC showed significant differences in peak coherence and average coherence between the conditions $(\mathrm{p}<0.05)$. Action observation application with FES in all frequency band showed the highest peak and average coherence value.

Conclusions: The results of this study are assumed to be the combination of increased eccentric information transfer from the sensorymotor cortex by action observation and an increased in concentric sensory input from the peripheral by the FES, suggesting that these are reflecting the sensorimotor integration process.

Keywords: Electroencephalography, Observation, Electromyography, Electric stimulation therapy, Coherence

\section{INTRODUCTION}

Central nervous system damage can cause disability, such as walking impairment, postural control, and muscle strength decreased sensory-motor performance. ${ }^{1,2}$ Therefore motor function recovery, especially the upper extremity function recovery, which is important for independent daily life, is an important goal of the central nervous system rehabilitation. ${ }^{3}$ The adult brain can adapt to environmental challenges, such as learning new skills, and to dysfunction caused by lesions on the central nervous system. ${ }^{4}$ It is driven by cortex activation, resulting in muscle activation with feedback from sensory receptors activated by movements. ${ }^{4}$ Recently, action observation has been studied as an intervention method for cognitive aspect supplementation, which is suggested to promote functional recovery after central nervous system injury. ${ }^{5,6}$ Until now, several studies reported that sensory-motor cortex activated during action execution can cause the

Received Nov 17, 2020 Revised Dec 12, 2020

Accepted Dec 17, 2020

Corresponding author Jiwon Park

E-mail mylovept@hanmail.net same pattern of action observation change. .11 $^{7-11}$

Functional electrical stimulation (FES) was introduced as a method, which artificially activates the sensory-motor system after a central nervous system injury, ${ }^{2}$ provide selectrical stimulation to the muscle or nerve for functional improvement, and has been reported to increase upper and lower limb functions. ${ }^{3,12-15}$ FES restores functional abilities and may also cause cortical excitability or brain plasticity changes. ${ }^{2,16}$ However, according to some studies, achieving motor function improvement using electrical stimulation alone in patients who lack active movement is difficult, though could be strengthened when cognitive and physical factors are properly provided. ${ }^{3}$

In recent years, several studies tried to investigate the correlation between brain waves and movements ${ }^{17-20}$ through the electrophysiological changes associated with motor control found in the central and peripheral nervous system levels by electroencephalogram (EEG) and electromyo-

Copylight (C)2020 The Korean Society of Physical Therapy

This is an Open Access article distribute under the terms of the Creative Commons Attribution Non-commercial License (https:// creativecommons.org/license/by-nc/4.0.) which permits unrestricted non-commercial use, distribution, and reproduction in any medium, provided the original work is properly cited. 
gram (EMG), or in the form of corticomuscular coherence (CMC). ${ }^{21} \mathrm{CMC}$ is the amplitude change of motor cortex oscillations during movement. Frequency coupling between brain oscillation and muscle rhythm has been observed during muscle activation, ${ }^{22} \mathrm{CMC}$ measures the degree of synchronisation between the oscillatory activity of the sensorimotor cortex and muscle. ${ }^{21}$ Although the physiological basis of CMC is not clear, it is now generally accepted that it reflects the communication between the motor cortex and motor units, ${ }^{23-25}$ showing that $\mathrm{CMC}$ reflects the connection and relationship between the central and peripheral activity. ${ }^{26}$

Combined physical and cognitive interventions facilitate motor recovery. ${ }^{3,4,27}$ Unfortunately, however, several studies separately used action observation and FES to activate damaged motor cortex, ${ }^{4,16,20,28,29}$ few studies have analyzed neurophysiological changes by combining action observation and FES. Therefore, in the present study, we tried to investigate the effects of action observation with FES on the communication between motor cortex and muscle through CMC analysis.

\section{METHODS}

\section{Subjects}

A total of twenty-seven healthy, nonathlete subjects participated in the study. The mean age \pm standard deviation was $25 \pm 3$ years. They are all right-handed based on the Edinburgh Handedness Inventory, had normal or corrected normal vision, had no neurological diseases, had not taken medicine for therapeutic purposes. All the experiments were conducted in a private laboratory with quiet rooms. All subjects gave informed written consent, and participated according to the Declaration of Helsinki, conducted after receiving an IRB deliberation from Daegu Catholic University, approval number is CUIRB-2016-0038.

\section{Experimental methods}

\section{1) EMG}

Surface electrodes (Delsys Trigno Wireless EMG system, Delsys Inc., Boston, MA, USA) were used to stimulate and record EMG activity and were attached at the proximal part at $2 \mathrm{~cm}$ away from the muscle belly of the wrist extensor and parallel to the running direction of the muscle fiber. The EMG signals were sampled at 2,000 Hz, bandpass filtered at 10-500 $\mathrm{Hz}$, resampled at $200 \mathrm{~Hz}$, stored on a personal computer for offline analysis, and rectified before coherence analysis. There were 1,000 data collected every 5 seconds, with a total of 60-70 segments used for the analysis.

\section{2) $E E G$}

Scalp EEG was recorded using a multichannel recorder (Neurofax EEG1,200, Nihon Kohden, Tokyo, Japan). Ag/AgCl ring electrodes filled with electroconductive gel were attached according to the international 10-20 electrode system. In this study, it was attached to $\mathrm{Nz}, \mathrm{Al}, \mathrm{A} 2, \mathrm{Fpl}, \mathrm{Fp} 2, \mathrm{Cz}$, C4, and C3 and to the peripheral electrodes C1, C5, FC3, and PC3 of C3 (Figure 1). Because analysis was performed only for the right hand in this study, only data from the $\mathrm{C} 3$ region were used. The EEG was calculated by taking the difference between the potentials at the $\mathrm{C} 3$ electrode and the mean of the four nearest neighbor electrodes (C1, C5, FC3, PC3) by a Laplacian algorithm. ${ }^{19,28,30}$ EEG recording was initiated when stable EEG was sustained without the artifacts, initiating all stimuli after 60 seconds. Electrode impedance was kept below $10 \mathrm{k} \Omega$. The lower and upper filters were set at $0.53 \mathrm{~Hz}$ and $60 \mathrm{~Hz}$, respectively, with a $200 \mathrm{~Hz}$ sampling rate, while notch filter was kept at $60 \mathrm{~Hz}$, avoiding power line interferences. All signals were A/D digitized and transferred using biosignal amplifier (NeuropackMEB-2200, Nihon-Koden, Japan) onto a PC for analysis. All segmented EEG data were inspected visually. Trials with eye blinks or other signal artifacts were excluded. The raw data was extracted every 5 seconds except for the first 1 second and the last 1 second.

\section{3) FES}

FES was performed with a Microstim (MedelGmBH, Berlin, Germany). Two electrodes were located on the forearm near the motor points of the extensor muscles of the wrist, with stimulation frequency at $30 \mathrm{~Hz}$ using a 300 ms biphasic constant current pulse with individual stimulation. ${ }^{28}$ The

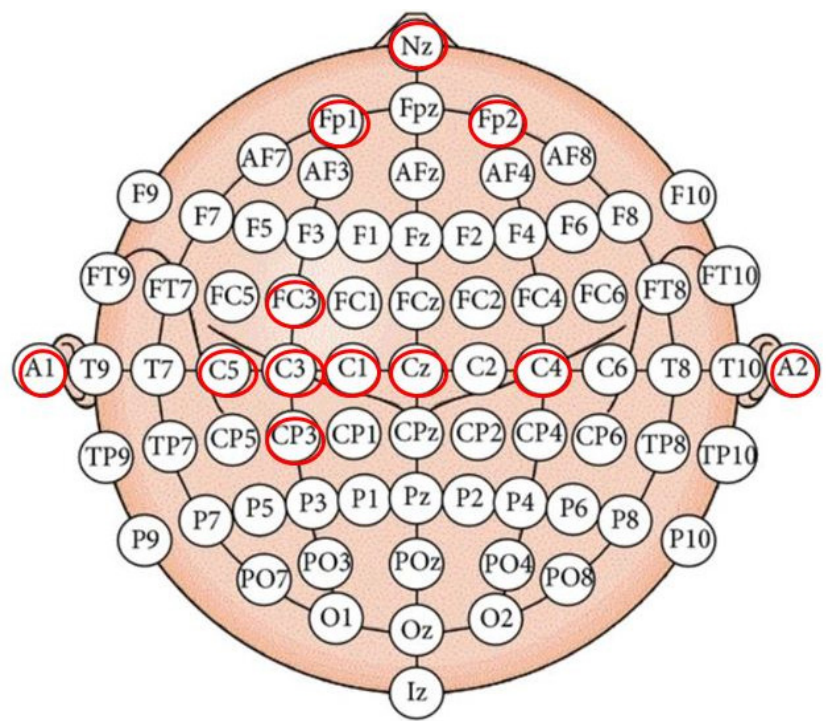

Figure 1. Attachment site of electrode. 
intensity was between $12 \mathrm{~mA}$ and $20 \mathrm{~mA}$, which was similar to the wrist extension angle at which the subject performed a comfortable motion by pretest.

\section{4) Action observation}

A 27-inch monitor approximately $100 \mathrm{~cm}$ in front of the subject featuring a cup being grabbed set at a slower speed than the actual movement provided the visual stimuli. During observation under FES condition, they were instructed to imagine the wrist movement, especially focusing on wrist extension for grabbing the cup and provided only white cross on black background and instructed not to imagine.

\section{5) Experimental paradigm}

In a dimly lit room, all subjects were seated in a comfortable chair with one's hand on the desk with elbow joint flexion and forearm pronation. They were leaning on the chair to get the most comfortable position, preventing unnecessary movements other than wrist movements. EEG and EMG were measured during action observation, FES, and action observation with FES for 7 seconds each session for 10 times. All randomized controlled trials were repeated for 30 times.

\section{6) Data analysis}

Simultaneously measured EEG raw data and rectified EMG signals were used to calculate cortical-muscular coherence, analyzing every 1,000 data collected using MATLAB software (MathWorks, US). The calculation procedures of coherence between two signals have been described in accordance to Halliday et al., ${ }^{5,251}$

$$
\operatorname{cohxy}(f)=|\operatorname{Rxy}(f)|^{2}=\frac{\left|P_{x y}(f)\right|^{2}}{P_{x x}(f) P y y(f)}
$$

where Pxy is the cross power spectrum for the EEG signal $(x)$ and the rectified EMG signal $(y)$ at a given frequency bin $(f)$ and $\mathrm{P}_{x x}$ and Pyy are the respective power spectrums for the EEG and EMG signals at the same frequency. ${ }^{31}$ Coherence greater than the $95 \%$ confidence limit (CL) was considered significant and computed as follows. ${ }^{24}$ In this study, only values above the calculated CL of 0.0306were used for the statistical analysis.

$$
C L(\alpha=0.95)=\frac{1-(1-\alpha) 1}{(L-1)}
$$

where $\alpha$ is the $95 \%$ significance level and $\mathrm{L}$ is the number of windows used for spectral estimation. CMC were divided into three frequency domains (alpha $8-13 \mathrm{~Hz}$, beta $14-30 \mathrm{~Hz}$, and gamma $31-50 \mathrm{~Hz}$ ), and the grand average coherence and peak coherence were computed. Repeated ANOVA was performed to analyze the difference in coherence values for each frequency band flowed by each condition. All analyses used the statistical analysis program SPSS for Windows 18.0, the bonferroni test was used as a post test, having a statistical significance level p of 0.05 .

\section{RESULTS}

Alpha, beta, and gamma CMCs showed significant differences in peak coherence and average coherence between the conditions $(\mathrm{p}<0.05)$ (Table 1). Post hoc test using the Bonferroni method showed significant difference in action observation conditions only, FES only, and action observa-

Table 1. Comparison of each band average coherence and peak coherence according to conditions

\begin{tabular}{|c|c|c|c|c|c|c|c|c|}
\hline & Ave CMC & $f$ & $p$ & Post.hoc & Peak CMC & $f$ & $p$ & Post.hoc \\
\hline \multicolumn{9}{|l|}{ Alpha } \\
\hline OBS & $0.00 \pm 0.00$ & 13.116 & $<0.001$ & $a / b / c$ & $0.01 \pm 0.01$ & 16.622 & $<0.001$ & $\mathrm{a} / \mathrm{b} / \mathrm{c}$ \\
\hline FES & $0.01 \pm 0.01$ & & & & $0.03 \pm 0.03$ & & & \\
\hline OBSFES & $0.03 \pm 0.03$ & & & & $0.05 \pm 0.05$ & & & \\
\hline \multicolumn{9}{|l|}{ Beta } \\
\hline OBS & $0.01 \pm 0.01$ & 27.237 & $<0.001$ & $a / b / c$ & $0.03 \pm 0.03$ & 24.060 & $<0.001$ & $a / b / c$ \\
\hline FES & $0.01 \pm 0.01$ & & & & $0.06 \pm 0.04$ & & & \\
\hline OBSFES & $0.03 \pm 0.02$ & & & & $0.15 \pm 0.12$ & & & \\
\hline \multicolumn{9}{|l|}{ Gamma } \\
\hline OBS & $0.01 \pm 0.00$ & 66.322 & $<0.001$ & $a / b / c$ & $0.03 \pm 0.04$ & 64.736 & $<0.001$ & $\mathrm{a} / \mathrm{b} / \mathrm{c}$ \\
\hline FES & $0.03 \pm 0.02$ & & & & $0.15 \pm 0.09$ & & & \\
\hline OBSFES & $0.06 \pm 0.03$ & & & & $0.22 \pm 0.08$ & & & \\
\hline
\end{tabular}

$(N=27)$

mean \pm standard deviation.

Ave CMC: average corticomuscular coherence, Peak CMC: peak corticomuscular coherence, OBS: only action observation condition, FES: only FES condition, OBSFES: action observation with FES condition. 

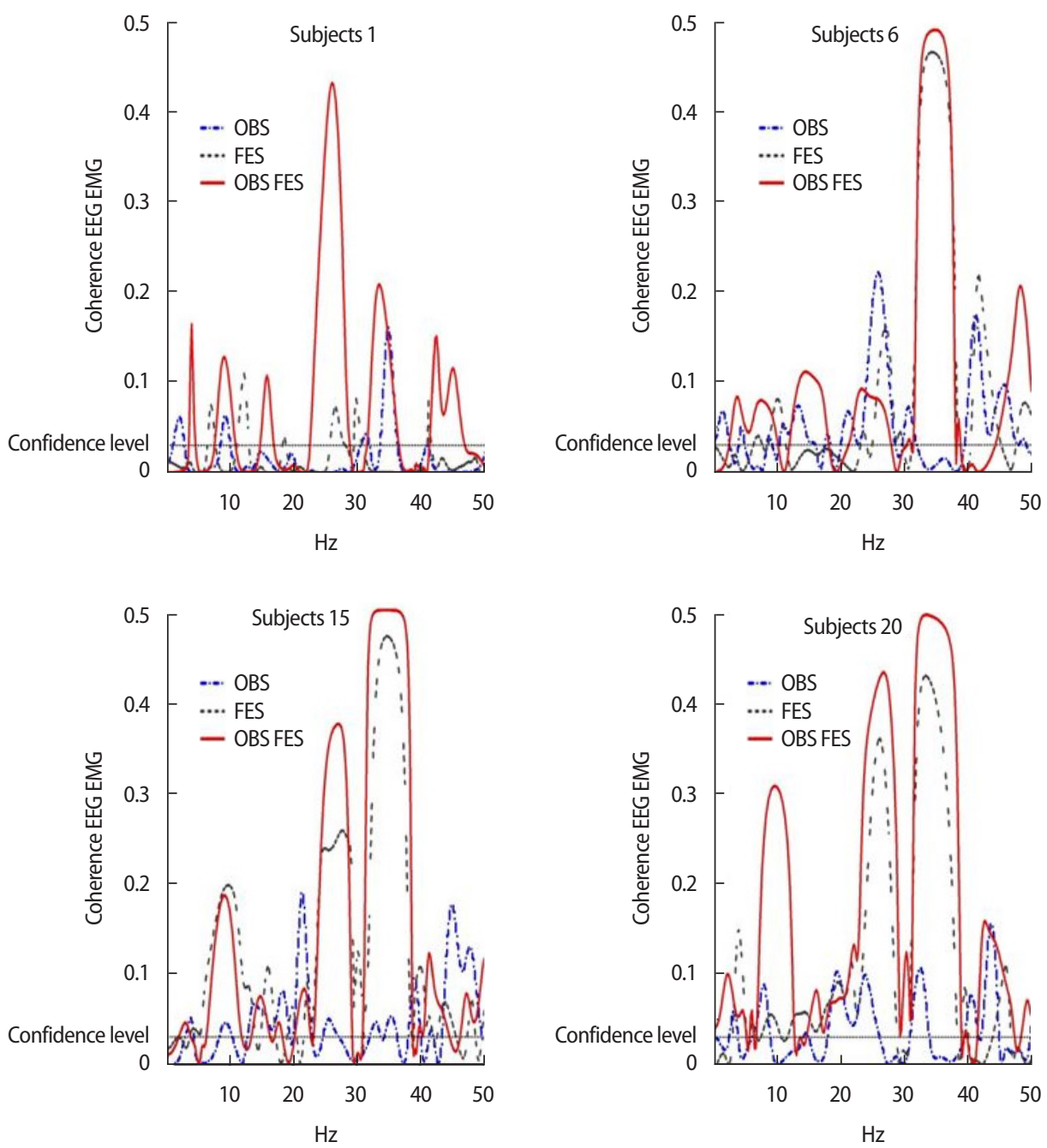

Figure 2. Changes of Corticomuscular coherence each condition.

tion with FES. The highest average and peak coherence values in alpha, beta, and gamma band were showed when action observation with FES is applied (Figure 2).

\section{DISCUSSION}

A number of studies tried to improve brain plasticity and induce functional improvements through various conditions. The purpose of this study was to investigate the effects of increased sensory input provided by FES on CMC during action observation. We analyzed the differences between providing action observation or FES alone and providing combination with FES during action observation. Dividing the highest and average CMC into frequency domain, each analysis result shows significant increase in all frequency domains when action observation with functional electric stimulation was applied simultaneously $(\mathrm{p}<0.05)$. Especially, the largest coherence was shown in the gamma band. We assumed that this result was a combination of the increased eccentric output from the sensorimotor cortex by action observation and increased concentric input from the peripheral muscle by the FES.

CMC represents an independent efferent phase adjustment process, working in parallel with cardinal motor control processing, ${ }^{21}$ related to the attention required to perform. ${ }^{32} \mathrm{McClelland}$ et al. ${ }^{32}$ suggested that the $\mathrm{CMC}$ frequency and magnitude may depend on motor cortex output and the afferent peripheral feedback. Junichi Ushiyama et al. ${ }^{33}$ suggested that the CMC mechanism may be a complex process involving feedback from the contracting muscle and sensorimotor cortex activity. Increased CMC in our study may be the result of overlapping from perceptual processing and motor control processing. In the study of Schoffelen et al. ${ }^{34}$ MagnetoEncephaloGrams (MEG) and EMG of the extensor carpi radialis muscle during wrist extension showed a spatial maximum CMC in the motor 
cortex contralateral to the muscle. Petersen et al. ${ }^{35}$ found that significant coupling between EEG and EMG during treadmill walking suggested a direct contribution of the motor cortex and corticospinal tract to muscle activity. Divekar and John ${ }^{21}$ tracked the recovery of the CMC. They found a significant decrease during hand and wrist immobilization and a significant increase when returned to pre-immobilization levels following recovery time. Moreover, Fang et al. ${ }^{26}$ found that functional corticomuscular connection is weakened during reaching after stroke.

In our knowledge, the study of alpha range CMC is few. Fang et al. ${ }^{26}$ and Mehrkanoon et al. ${ }^{36}$ suggested that alphaband coherence is considered to indicate communication between the brain and muscle, exposing the feedback and feedforward interactions associated with movement. Mat Safri et al. ${ }^{37}$ found that enhanced alpha waves in EEG recordings of subjects maintaining the same isometric contraction suggested to be associated with attentional motor processes. Petersen et al. ${ }^{35}$ found that alphaband coherence dominance during normal walking condition may reflect the afferent information inflow to the cortical network.

Beta range $\mathrm{CMC}$ is known to play an important role in sensorimotor integration, ${ }^{32}$ related to demand toward the motor task and attention, ${ }^{37}$ and associated with mechanism of maintaining the current sensorimotor state. ${ }^{36}$ Since numerous studies showed beta band oscillatory activity involved in motor control, ${ }^{21,23,32,34,38}$ we assumed that beta CMC increase is due to the communication between the sensory-motor cortex and the contralateral muscles. Yang et al. ${ }^{23}$ and David et al. ${ }^{17}$ reported a significant EEG-EMG coherence at beta frequency band during voluntary motor performance in healthy subjects. Monica et al. ${ }^{39}$ showed that a voluntary contraction controlled by one hemisphere can influence beta coherence contralaterally during the hand grip. Mat Safri et al. ${ }^{37}$ suggested that increased motor task by the visual stimuli can enhance CMC between the motor cortex and muscle. In Perez et al. ${ }^{25}$ study, a significant increase in beta coherence during visuomotor skill training is shown supposing that it reflects sensorimotor integration processes between the cortex and muscle. McClelland et al..$^{32}$ showed that electrical or mechanical peripheral stimuli produced the B-band CMC pattern modulation. Moreover, Jacobs et al. ${ }^{38}$ suggested that significant beta CMC is evident during human standing balance and responsive to mechanical changes. Divekar and $J \mathrm{ohn}^{21}$ assumed that beta CMC has higher motor output stability functional roles through improved sensorimotor integration, though it weakened during moderate isometric contractions ${ }^{21}$ and eventually disappeared during movement. ${ }^{36}$

Various studies reported that gamma band coherence can be altered by preparatory movements and cognitive factors while maintaining maximum contraction and dynamic voluntary movements, such as reaching for a target. ${ }^{23,26,37,40}$ Fang et al. ${ }^{26}$ suggested that gamma band muscle activities linearly correlate with focal activity in the contralateral motor cortex, which could be a fundamental feature of the motor system to organize voluntary movements. Gwin and Ferris ${ }^{24}$ and Mehrkanoon et al. ${ }^{36}$ suggested that gamma band coherence has been linked to the dynamic force output during movement preparation, which was found to be significantly greater for isotonic exercises than for isometric exercises. ${ }^{24}$ The highest gamma CMC in this study is assumed to be due to the cognitive factors by action observation and wrist movement induced by FES. Attention is known to increase gamma band activity in the somatosensory cortex.36 Gwin and Ferris ${ }^{24}$ suggested that the required integration of visual and somatosensory information may increase gamma coherence. Muthukumaraswamy ${ }^{22}$ showed that gamma coherence may occur during the production of dynamic movements, while gamma oscillations are mostly linked to movement production. Moreover, a previous study found that reduced gamma CMC in stroke patients compared to normal adults during reaching task suggested a poor brain-muscle communication or poor integration of the gamma band $\mathrm{CMC}$, reflecting the mechanism of movement defects after stroke. ${ }^{26}$

The results of this study showed that the simultaneous action observation with FES can increase CMC compared to action observation or FES alone, which is assumed to be the result of the combination of increased eccentric information transfer from the sensorimotor cortex by action observation and an increased concentric sensory input from the peripheral by the FES and may reflect the process of sensorimotor integration. The combination of action observation and FES in areas of neural rehabilitation will promote brain plasticity and affect the reorganization with the corresponding segment muscle, which will have a positive effect on the functional improvement of the subject.

\section{REFERENCES}

1. Bauer P, Krewer C, Golaszewski S et al. Functional electrical stimulationassisted active cyclingtherapeutic effects in patients with hemiparesis from 7 days to 6 months after stroke: A randomized controlled pilot study. Arch Phys Med Rehabil. 2015;96(2):188-96.

2. Popovic DB. Advances in functional electrical stimulation (FES). J Electromyogr Kinesiol. 2014;24(6):795-802.

3. Kim HJ, Lee GC, Song CH. Effect of functional electrical stimulation with mirror therapy on upper extremity motor function in poststroke patients. J Stroke and Cerebrovasc Dis. 2014;23(4):655-61. 
4. Reynolds C, Osuagwu BA, Vuckovic A. Influence of motor imagination on cortical activation during functional electrical stimulation. Clin Neurophysiol. 2015;126(7):1360-9.

5. Halliday D, Rosenberg J, Amjad A et al. A framework for the analysis of mixed time series/point process data theory and application to the study of physiological tremor, single motor unit discharges and electromyograms. Prog Biophys Mol Biol. 1995;64(2):237-78.

6. Tia B, Mourey F, Ballay Y et al. Improvement of motor performance by observational training in elderly people. Neurosci Lett. 2010;480(2):13842.

7. Oberman LM, Hubbard EM, McCleery JP et al. EEG evidence for mirror neuron dysfunction in autism spectrum disorders. Cognitive Brain Research. 2005;24(2):190-8.

8. Bernier R, Dawson G, Webb S et al. EEG mu rhythm and imitation impairments in individuals with autism spectrum disorder. Brain Cognition. 2007;64(3):228-37.

9. Pineda JA. The functional significance of mu rhythms: Translating "seeing" and "hearing" into "doing". Brain Res Rev. 2005;50(1):57-68.

10. Babiloni C, Babiloni F, Carducci F et al. Human cortical electroencephalography (EEG) rhythms during the observation of simple aimless movements: A high-resolution EEG study. NeuroImage. 2002;17(2): $559-72$.

11. Puzzo I, Cooper NR, Cantarella S et al. Measuring the effects of manipulating stimulus presentation time on sensorimotor alpha and low beta reactivity during hand movement observation. NeuroImage. 2011;57(4): 1358-63.

12. Monaghan CC, Hermens HJ, Nene AV et al. The effect of FES of the tibial nerve on physiological activation of leg muscles during gait. Med Eng Phys. 2010;32(4):332-8.

13. Street T, Taylor P, Swain I. Effectiveness of functional electrical stimulation on walking speed, functional walking category, and clinically meaningful changes for people with multiple sclerosis. Arch Phys Med Rehabil. 2015;96(4):667-72.

14. Springer S, Vatine JJ, Wolf A et al. The effects of dual-channel functional electrical stimulation on stance phase sagittal kinematics in patients with hemiparesis. J Electromyogr Kinesiol. 2013;23(2):476-82.

15. Cho MK, Kim JH, Chung Y et al. Treadmill gait training combined with functional electrical stimulation on hip abductor and ankle dorsiflexor muscles for chronic hemiparesis. Gait Posture. 2015;42(1):73-8.

16. Shendkar CV, Lenka PK, Biswas A et al. Therapeutic effects of functional electrical stimulation on gait, motor recovery, and motor cortex in stroke survivors. Hong Kong Physiother J. 2015;33(1):10-20.

17. HallidayDM, Conway BA, Farmer SF et al. Using electroencephalography to study functional coupling between cortical activity and electromyograms during voluntary contractions in humans. Neurosci Lett. 1998;271:5-8.

18. Gu Y, Dremstrup K, Farina D. Single-trial discrimination of type and speed of wrist movements from EEG recordings. Clin Neurophysiol. 2009;120(8):1596-600.

19. Houdayer E, Labyt E, Cassim F et al. Relationship between event-related beta synchronization and afferent inputs: Analysis of finger movement and peripheral nerve stimulations. Clin Neurophysiol. 2006;117(3):62836.

20. Mouthon A, Ruffieux J, Walchli M et al. Task-dependent changes of cor- ticospinal excitability during observation and motor imagery of balance tasks. Neuroscience. 2015;303:535-43.

21. Divekar NV, John LR. Neurophysiological, behavioural and perceptual differences between wrist flexion and extension related to sensorimotor monitoring as shown by corticomuscular coherence. Clin Neurophysiol. 2013;124(1):136-47.

22. Muthukumaraswamy SD. Temporal dynamics of primary motor cortex gamma oscillation amplitude and piper corticomuscular coherence changes during motor control. Exp Brain Res. 2011;212(4):623-33.

23. Yang Q, Fang Y, Sun CK et al. Weakening of functional corticomuscular coupling during muscle fatigue. Brain Res. 2009;1250:101-12.

24. Gwin JT, Ferris DP. Beta and gamma-range human lower limb corticomuscular coherence. Front Hum Neurosci. 2012;6:258.

25. Perez MA, Lundbye-Jensen J, Nielsen JB. Changes in corticospinal drive to spinal motoneurones following visuo-motor skill learning in humans. J Physiol. 2006;573(3):843-55.

26. Fang Y, Daly JJ, Sun J et al. Functional corticomuscular connection during reaching is weakened following stroke. Clin Neurophysiol. 2009;120 (5):994-1002.

27. Park CS, Kang KY. The effects of additional action observational training for functional electrical stimulation treatment on weight bearing, stability and gait velocity of hemiplegic patients. J Phys Ther Sci. 2013;25(9): 1173-5.

28. Müller GR, Neuper C, Rupp R et al. Event-related beta EEG changes during wrist movements induced by functional electrical stimulation of forearm muscles in man. Neurosci Lett. 2003;340(2):143-7.

29. Tanaka M, Kubota S, Onmyoji Y et al. Effect of tactile stimulation on primary motor cortex excitability during action observation combined with motor imagery. Neurosci Lett. 2015;600:1-5.

30. Yasunari Hashimoto, Junichi Ushiba, Akio Kimura et al. Correlation between EEG-EMG coherence during isometric contraction and its imaginary execution. Acta Neurobiol Exp. 2010;70(1):76-85.

31. Johnson AN, Wheaton LA, Shinohara M. Attenuation of corticomuscular coherence with additional motor or non-motor task. Clin Neurophysiol. 2011;122(2):356-63.

32. McClelland VM, Cvetkovic Z, Mills KR. Modulation of corticomuscular coherence by peripheral stimuli. Exp Brain Res. 2012;219(2):275-92.

33. Junichi Ushiyama, Yuji Takahashi, Ushiba J. Muscle dependency of corticomuscular coherence in upper and lower limb muscles and trainingrelated alterations in ballet dancers and weightlifters. J Appl Physiol. 2010;109(4):1086-95.

34. Schoffelen JM, Oostenveld R, Fries P. Imaging the human motor system' s beta-band synchronization during isometric contraction. Neuroimage. 2008;41(2):437-47.

35. Petersen TH, Willerslev-Olsen M, Conway BA et al. The motor cortex drives the muscles during walking in human subjects. J Physiol. 2012; 590(10):2443-52.

36. Mehrkanoon S, Breakspear M, Boonstra TW. The reorganization of corticomuscular coherence during a transition between sensorimotor states. Neuroimage. 2014;100:692-702.

37. Mat Safri N, Murayama N, Hayashida Y et al. Effects of concurrent visual tasks on cortico-muscular synchronization in humans. Brain Res. 2007; 1155:81-92.

38. Jacobs JV, Wu G, Kelly KM. Evidence for beta corticomuscular coher- 
ence during human standing balance: Effects of stance width, vision, and support surface. Neuroscience. 2015;298:1-11.

39. Perez MA, Soteropoulos DS, Baker SN. Corticomuscular coherence during bilateral isometric arm voluntary activity in healthy humans. J
Neurophysiol. 2012;107(8):2154-62.

40. Schoffelen JM, Poort J, Oostenveld R et al. Selective movement preparation is subserved by selective increases in corticomuscular gamma-band coherence. J Neurosci. 2011;31(18):6750-8. 